\title{
Femtosecond Laser Writing of Optical Waveguides by Self-Induced Multiple Refocusing in $\mathrm{LiTaO}_{3}$ Crystal
}

\author{
Lingqi Li ${ }^{\circledR}$, Weijie Nie, Ziqi Li ${ }^{\circledR}$, Bin Zhang ${ }^{\circledR}$, Lei Wang ${ }^{\circledR}$, Patricia Haro-González ${ }^{\circledR}$, Daniel Jaque, \\ Javier R. Vázquez de Aldana, and Feng Chen ${ }^{\circledR}$, Senior Member, OSA
}

\begin{abstract}
We report on a one-step fabrication method of horizontal dual-line waveguides supporting TE guidance in $\mathrm{LiTaO}_{3}$ crystal utilizing multiple refocusing mechanisms of the femtosecond laser. Through the nonlinear process, multiple foci can be formed spontaneously with the interval of a few micrometers along the laser propagation direction. The number and the separation of focal spots, even the entire length of laser-induced tracks, could be modulated by varying the laser parameters. Taking advantage of this spontaneous phenomenon, we further implemented the multiscan technique to write two parallel damage lines along transverse direction, realizing stress-induced waveguides by horizontal light confinement. Moreover, using this method, one-step two-channel guidance could also be demonstrated by adjusting the pulse energy. The fabrication details and waveguiding performances were studied by both experimental and computational methods. This paper opens the alternative way to construct complex integrated platforms in $\mathrm{LiTaO}_{3}$ crystal by using femtosecond laser writing.
\end{abstract}

Index Terms-Laser materials processing, lithium tantalate, nonlinear optics, optical waveguides.

\section{INTRODUCTION}

$\mathbf{O}$ PTICAL waveguides fabricated by femtosecond laser writing provide a miniaturized platform for versatile integrated photonic devices, in which could confine light propagation within dimensions of micrometric or sub-micrometric scales [1]. Therefore, the optical features of nonlinear responses

Manuscript received October 29, 2018; revised April 14, 2019; accepted May 12, 2019. Date of publication May 15, 2019; date of current version June 24 , 2019. This work was supported in part by the National Natural Science Foundation of China under Grant 61775120 and Grant 11874239, in part by the Ministerio de Economía y Competitividad under Grant FIS2017-87970-R, in part by the Ministerio de Economía y Competitividad de España under Grant MAT2016-75362-C3-1-R, and in part by the Autónoma de Madrid under Grant B2017/BMD-3867RENIM-CM. (Corresponding author: Feng Chen.)

L. Li, W. Nie, Z. Li, B. Zhang, L. Wang, and F. Chen are with the School of Physics and the State Key Laboratory of Crystal Materials, Shandong University, Jinan 250100, China (e-mail: lingqiLee@163.com; weijienie@163.com; drziqili@163.com; phybinzhang@163.com; sduwl@163.com; drfchen@sdu. edu.cn).

P. Haro-González and D. Jaque are with the Fluorescence Imaging Group, Departamento de Física de Materiales, Facultad de Ciencias, Universidad Autónoma de Madrid, 28049 Madrid, Spain (e-mail: haroglez@gmail.com; daniel.jaque@uam.es).

J. R. Vázquez de Aldana is with the Aplicaciones del Láser y Fotónica Group, Departamento de Física, Universidad de Salamanca, 37008 Salamanca, Spain (e-mail: jrval@usal.es).

Color versions of one or more of the figures in this paper are available online at http://ieeexplore.ieee.org.

Digital Object Identifier 10.1109/JLT.2019.2917076 and lasing performances, could be enhanced in various waveguiding structures [2]-[7]. In recent years, femtosecond laser writing has been consolidated as a powerful technique to fabricate various three-dimensional microstructures in diverse transparent materials [8]-[11]. For large laser powers, severe damage is produced with a refractive index decrease in the laser-induced tracks (the so-called Type-II material modification). However, due to the stress induced during the laser-matter interaction, a refractive index increase is created in the vicinity of the damage lines, that can be used for waveguide fabrication usually constructed with two parallel damage tracks (dual-line technique) in order to improve confinement. Such waveguides seem to be more advantageous compared with the standard direct writing geometry based on weak material modifications (Type-I) in which the guiding core is located at the damaged region [12] Firstly, the dual-line waveguides preserve well the luminescence and nonlinear properties of the bulk material. And secondly, the stressinduced waveguides are stable and even not removed under highpower applications [13].

However, the configuration of dual-line usually elongate along the propagation direction of laser pulse (longitudinal direction), thus, only TM polarization is well guided for dual-line waveguides in some cubic crystals like Nd:YAG [2], or even trigonal crystals like $\mathrm{LiNbO}_{3}$ [3] and $\mathrm{LiTaO}_{3}$ [14]. As a result, achieving horizontal dual-line geometry supporting TE polarization is more challenging as the sample is translated perpendicular to the laser beam, but is further crucial to achieve 2D guidance. For this purpose, there is a method to fabricate two parallel lines oriented in horizontal direction supporting TE polarization via a shaped femtosecond laser pulse by a spatial light modulator, nevertheless, with the drawback of the roughness of the laser-induced tracks at cross-sections [15]. With this motivation, more practical fabrication methods to achieve guiding confinement of horizontal polarization is desirable.

In our work, we introduce a novel method to fabricate such waveguides making use of the complex non-linear dynamics of ultrashort pulse propagation. With short pulses, high peak intensities are achieved in a small volume with modest energy per pulse [16]. Therefore, the dielectric medium is readily driven into a nonlinear regime, dynamically changing the temporal, spatial, and spectral properties of the laser pulse, leading to spectacular new effects [17]. For instance, self-focusing occurs due to a non-permanent local refractive index change 
self-induced by the laser field because of the 3rd-order nonlinearity (Kerr nonlinearity) of the medium when the laser power exceeds a critical power $P_{c r}$, which may lead to catastrophic collapse of the beam. This process, together with plasma generation by nonlinear photoionization, results in a continual localization of a high-intensity spot of the pulse along the propagation direction [18]-[21]. As a consequence, these nonlinear processes have often been regarded as a problem to be avoided due to the existence of undesirable effects and a distortion of the waveguide shape [22]. However, self-focusing of femtosecond lasers could also be demonstrated an easy and flexible method for producing periodic sub-micron and nano-scale structures used as photonic elements such as photonic crystals and microgratings. Up to now, the self-fabrication of void array by femtosecond laser irradiation has been reported in fused silica, borosilicate glass, BK7 glass and PMMA [23]-[26]. Compared with these materials, optical crystals are important platforms for very different applications. However, the spontaneous phenomenon has been reported only in sapphire $\left(\mathrm{Al}_{2} \mathrm{O}_{3}\right), \mathrm{CaF}_{2}$ and $\mathrm{SrTiO}_{3}$ crystals [27]-[29]. It is valuable to explore the spontaneous phenomenon in various crystals to realize the potential applications.

Lithium tantalate $\left(\mathrm{LiTaO}_{3}\right)$ is a well-known multifunctional crystal belongs to the trigonal $3 \mathrm{~m}\left(\mathrm{C}_{3 v}\right)$ crystallographic point group similar to $\mathrm{LiNbO}_{3}$, which has wide nonlinear applications in optical parametric amplifiers and generators, frequency doubles because of a high $\chi_{33}$ nonlinear coefficient, a shorter UV absorption edge value $(0.26 \mu \mathrm{m})$ and a low coercive field for ferroelectric domain inversion [30], [31].

In this work, we report a novel strategy to fabricate horizontal dual-line waveguides supporting TE-polarized light taking advantage of multiple refocusing mechanisms. Our interest is in applying these multiple foci formed spontaneously by the nonlinear dynamics of ultrashort pulse propagation, assisted with multi-scan technique to write two or multiple parallel lines oriented in the transverse direction, realizing horizontal light confinement. Moreover, two-channel guidance consisted with three parallel lines would also be demonstrated by using this method. The dependence of the foci number and the interval between two consecutive spots on the laser parameters, including pulse energy and focal depth has been investigated. The influence of laser writing parameters (pulse energy and focal depth) on the guiding properties and modal profiles of the waveguides have been characterized.

\section{EXPERIMENTS IN DETAILS}

Fig. 1 depicts the schematic fs-laser-writing process of horizontal-polarization waveguide in $\mathrm{LiTaO}_{3}$ crystal. The $z$-cut $1 \mathrm{~mol} \% \mathrm{MgO}$ : stoichiometric $\mathrm{LiTaO}_{3}$ crystal (MgSLT) sample used in this work was cut into wafers with dimensions of $0.5(z)$ $\times 5(y) \times 10(\times) \mathrm{mm}^{3}$. During the laser writing process, an optical fiber laser system (Origami-10 XP, OneFive) was utilized, in which linearly polarized pulses ( $420 \mathrm{fs}$ duration, $1031 \mathrm{~nm}$ central wavelength, $50 \mu \mathrm{J}$ maximum energy, and $10 \mathrm{kHz}$ repetition rate) were generated. The pulse energy was controlled by a rotatable half-wave plate and a Glan-Taylor polarizer. The femtosecond

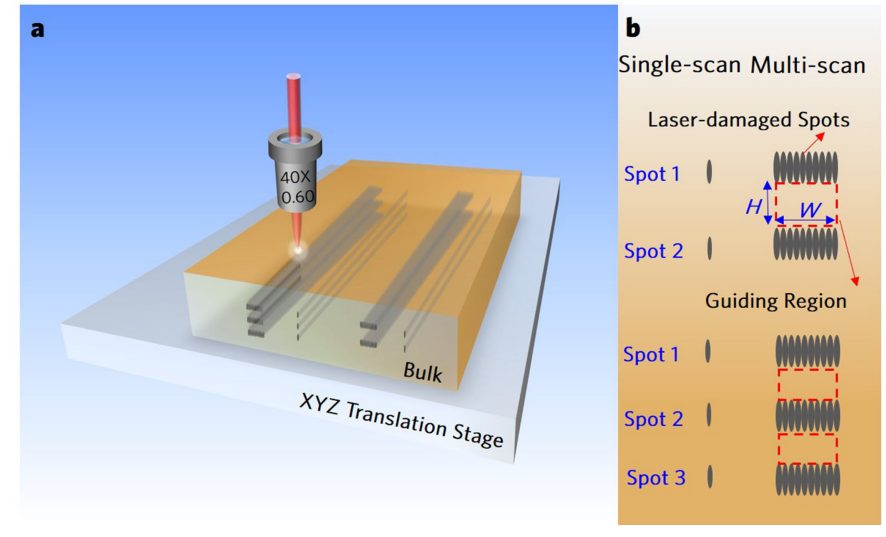

Fig. 1. The fabrication of horizontal-polarization optical waveguides in MgSLT. (a) Schematic plot of the fabrication process with the femtosecond laser. (b) The schematic diagram of multiple foci modification and configuration of single-channel and two-channel optical waveguides.

laser beam was focused $150 \mu \mathrm{m}$ below the largest surface $(5 \mathrm{~mm}$ $\times 10 \mathrm{~mm}$ ) by a $40 \mathrm{X}$ microscope objective $(\mathrm{NA}=0.60)$. During the writing process, the sample was placed at a $3 \mathrm{D}$ motorized stage with a spatial resolution of $0.1 \mu \mathrm{m}$. Then, the sample was scanned at a constant velocity of $4 \mathrm{~mm} / \mathrm{s}$. A damage line inside the sample was performed with the scanning direction parallel to the laser polarization which was aligned with the 10 -mm edge. Under this condition, many different pulse energies were carried out to vary the geometry of the damage microstructures.

A microscope (Axio Imager, Carl Zeiss) was utilized to photograph the cross sections of the horizontal-polarization waveguide in $\mathrm{LiTaO}_{3}$ crystal. To experimentally investigate the characterization of the waveguides, confocal $\mu$-Raman mapping imaging and guidance have been carried out. For the confocal $\mu$ Raman mapping analysis, a fiber coupled confocal microscope (Olympus BX-41) integrated with a single-mode 488-nm Argon laser is used as excitation source. The laser beam was set to a power of $30 \mathrm{~mW}$ and was focused onto the optical-grade polished surface of the sample with $50 \mathrm{X}$ MPlan $(\mathrm{NA}=0.55)$, microscope objective lens for micron resolution imaging, respectively. The back-scattered Raman signal was collected with the same lens and, after passing through a confocal aperture, was coupled into a $50 \mu \mathrm{m}$ core fiber connected to a high-resolution spectrometer. The sample was positioned on precision XY motorized stages with the written waveguide parallel to the analyzing beam such that a spatial Raman spectral map of the waveguide cross section was generated. The focus of the pumping laser was moved with a step of $1 \mu \mathrm{m}$ and the scattered Raman signal was along the z-axis of the MgSLT crystal. For the guiding performance of the different channel waveguides, a typical end-coupling system has been employed with a linearly polarized $\mathrm{He}-\mathrm{Ne}$ lasers at a wavelength of $633-\mathrm{nm}$. The laser was coupled into the end-face of the MgSLT sample by an objective lens $(\mathrm{NA}=0.40)$. Then, another identical objective lens was utilized at the output of the waveguide, and the modal profile was captured via CCD. A halfwave plate was used to change the polarization of input light. The polarization guiding properties were characterized. With the above arrangement, the propagation losses were determined by 


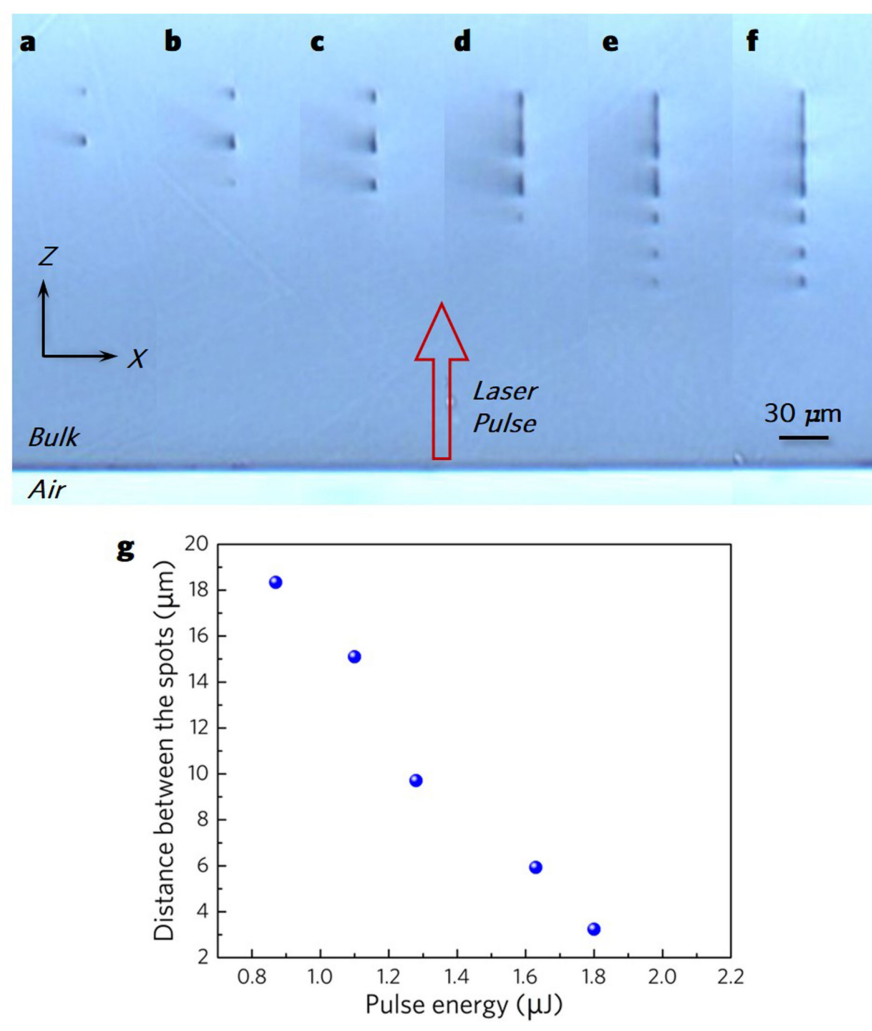

Fig. 2. Optical image of the cross sections of laser induced microstructures at pulse energies $0.87,1.10,1.28,1.63,1.80$, and $2.03 \mu \mathrm{J}$ from left to right, corresponding to (a)-(f), respectively. (g) Dependence of the distance between the spots on laser pulse energy with fixed focal depth at $150 \mu \mathrm{m}$.

directly measuring the light powers coupled into and out of the end-faces.

\section{RESULTS AND DISCUSSION}

We first investigated the influence of incident pulse energy on the generated spot array. Fig. 2(a)-(f) show the cross-sectional microscope images of aligned damage microstructures with different pulse energy at a fixed geometrical focal depth of $150 \mu \mathrm{m}$. The number of focal spots increases with increasing the pulse energy. The underlying physical mechanism of the multiple foci could be attributed to the dynamic competition between the selffocusing caused by the Kerr effect and the defocusing caused by the plasma generated [19].

The Kerr nonlinearity (the 3rd-order nonlinearity) results in an intensity-dependent refractive index change that follows the spatial intensity profile of the laser. Generally, the nonlinear refractive index $n_{2}$ of most materials is usually positive in the femtosecond laser direct-write regime, thus, this index change acts as a self-induced lens and causes the beam to focus [17]. This is predicted to occur as the peak power of the laser beam is above the critical power $P_{c r}$, given by [18].

$$
P_{\text {cr }}=\frac{3.77 \lambda^{2}}{8 \pi n_{0} n_{2}}
$$

where $\lambda$ is the central wavelength of the pulse, $n_{0}$ is the linear index of refraction, and $n_{2}$ is the coefficient of the Kerr

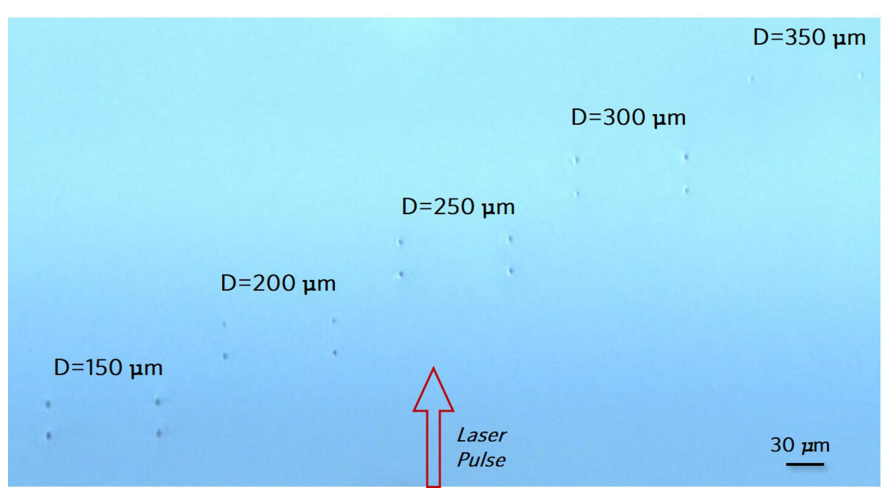

Fig. 3. Optical microscope image of double spots at different focal depths. Two tracks are written at one depth with same laser writing parameters. The pulse energy was fixed at $0.87 \mu \mathrm{J}$.

nonlinear index of refraction. In the case of MgSLT, the value of $P_{c r} \approx 0.82 \mathrm{MW}$, with the $n_{0}=2.13$ and $n_{2}=0.91 \times$ $10^{15} \mathrm{~cm}^{2} \mathrm{~W}^{-1}$ [32]. Peak power of the self-focused pulses is $P_{p}=0.88 E_{p} / \tau, E_{p}$ is pulse energy and $\tau$ is pulse width. The value of $P_{c r}$ corresponds to a pulse energy of $0.3 \mu \mathrm{J}$ and the minimal peak power used in our work was $P \approx 3 P_{c r}$, Thus, the phenomenon of multiple foci is expected to occur due to the energy excess above the threshold. As the laser beam selffocuses, the intensity increases giving rise to the generation of free electrons through strong-field photoionization processes. The generated plasma modifies itself the refractive index, acting as a defocusing lens that competes with the self-focusing effect. The cycle of focusing-defocusing repeats as peak power is higher than the threshold power of self-focusing [20]. As a result, the damaged areas consist of several point-like structures where the pulse has been strongly focused, or longer structures where self-focusing and plasma defocusing are nearly compensated (filamentation) [33].

In order to understand the relationship between the distance of two consecutive spots and the laser pulse energy, we plotted the interval of the spots as a function of the pulse energy in Fig. 2(g). The average interval of two neighboring spots in each damage track is determined by the position of the highest peak in its Fourier spectrum. As can be seen in Fig. 2(g), the interval between the spots decreases with the increasing pulse energy. This phenomenon agrees well with the result in fused silica and BK7 glass [25]. This is probably because the higher input energy of pulse, the shorter the cycle of beam oscillation, and the shorter distance between the spots [20].

We then investigated the influence of the focal depth beneath the surface on the induced spots array by moving the crystal along the propagation direction of the laser beam at seven different focal depths ( $100 \mu \mathrm{m}, 150 \mu \mathrm{m}, 200 \mu \mathrm{m}, 250 \mu \mathrm{m}, 300 \mu \mathrm{m}$, $350 \mu \mathrm{m}$ and $400 \mu \mathrm{m}$ ). Fig. 3 shows the microscope image of the spots array with $0.87 \mu \mathrm{J}$ pulse energy at various depths. It is observed that there is no detectable damage spot at depth of $100 \mu \mathrm{m}$ and $400 \mu \mathrm{m}$. Particularly, there is only one spot at the deeper depth of $350 \mu \mathrm{m}$. The multi-foci phenomenon is then 


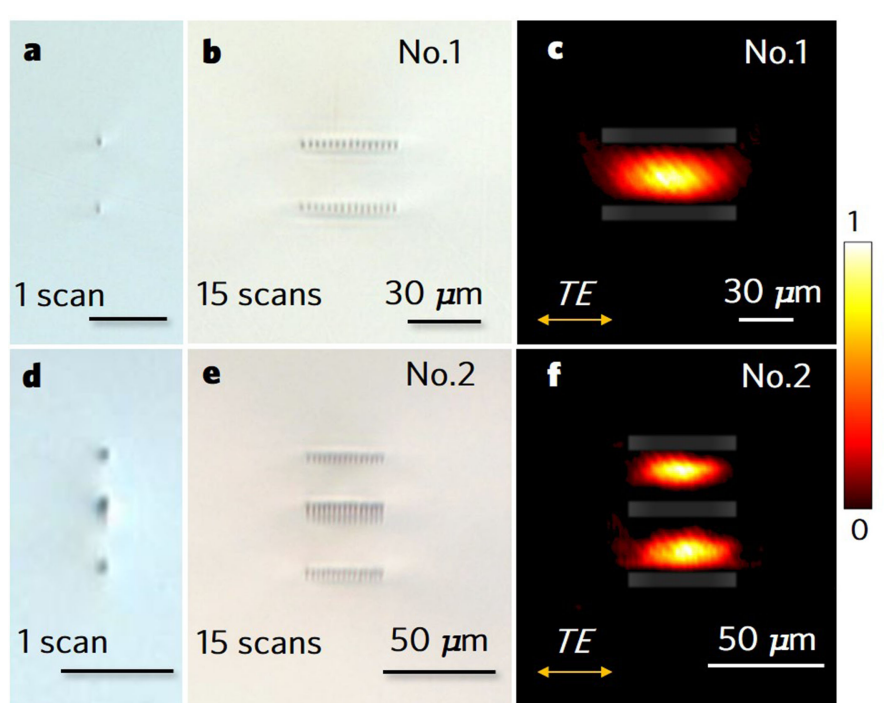

Fig. 4. The characterization of the horizontal-polarization optical waveguides in MgSLT. Optical microscope image of the cross section of multiple foci via femtosecond single-scan (a) and (d), horizontal-polarization waveguides by 15 scans, No.1, single-channel (b) and No. 2, two-channel (e). (c) and (f) Measured near-field modal profiles of waveguides No. 1 and No. 2 for TE polarizations at $633 \mathrm{~nm}$

limited within a depth range of $150 \mu \mathrm{m}$ to $300 \mu \mathrm{m}$. This phenomenon is understood to be as follows: for the shallower focal depth, the propagation distance in the crystal is not large enough to induce self-focusing in the pulse, so that there is no observation of damage tracks when focusing near the surface $(100 \mu \mathrm{m})$ [34]. For the deeper focal depth, two effects may contribute to decrease the peak intensity of the pulse, and consequently to suppress damage: on the one hand, the linear dispersion of the pulse that makes the pulse duration to increase with propagation in the sample [28], and, on the other hand, the spherical aberration (degradation of the focal spot due to refraction at the air-crystal boundary) whose effect increases with the focal depth, thus lowering the peak power of the pulse [29]. When the peak power becomes smaller than the $P_{c r}$, the self-focusing would not happen, as a result, there is only one focal point. In addition, the interval between the two spots increases slightly from $17.59 \mu \mathrm{m}$ to $19.22 \mu \mathrm{m}$ with the increase of focal depth, which could be explained by the decreased pulse peak power. The lower the input peak power, the longer the cycle of beam oscillation, and the longer distance between two spots [20].

In order to construct horizontal dual-line waveguides supporting TE guidance, we used the two and three damage spots (Fig. 4(a) and (d)) inscribed by laser single-scan, further implemented the multi-scan technique to write two parallel damage lines along transverse direction. The pulse energy was $0.87 \mu \mathrm{J}$ and $1.12 \mu \mathrm{J}$, corresponding to waveguides No.1 and No.2, respectively. The focal depth is $150 \mu \mathrm{m}$. Fig. 4(b) demonstrates the microscope images of horizontal dual-line optical waveguides in MgSLT via multi-scan procedure. Particularly, one-step twochannel writing has been realized as shown in Fig. 4(e). The laser-induced tracks show a smooth structure, contrasting to the roughness of tracks inscribed by a slit-beam shaping technique
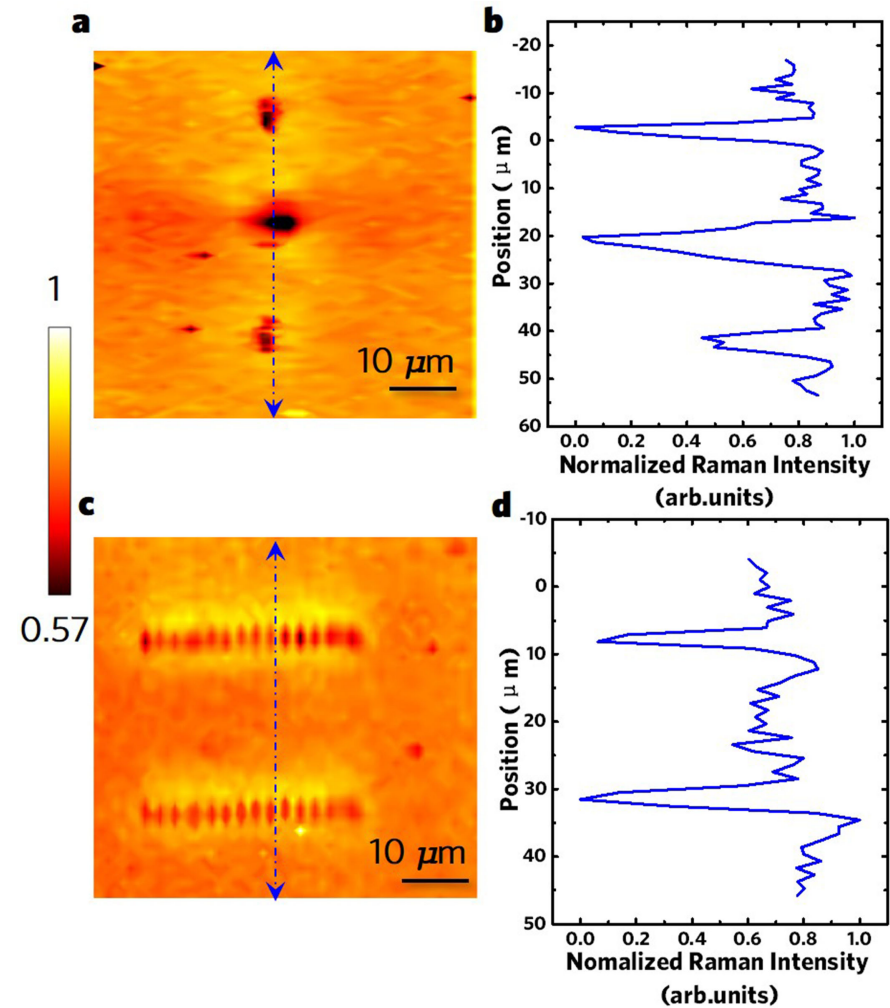

Fig. 5. Confocal Raman intensity spatial mapping of multiple foci modification (a) (top) and horizontal-polarization waveguide (bottom) in MgSLT. (b) and (d) Raman intensity profile obtained along the longitudinal scan direction depicted in (a) and (c).

via a spatial light modulator [15]. As expected, both waveguides (single-channel and two-channel) have shown better guidance along the TE polarization as exhibited in Fig. 4(c) and (d), whereas the standard Type II waveguides reported before in trigonal crystals only supported guidance along TM polarization.

To obtain additional evidence supporting the waveguide morphology, we have also performed $\mu$-Raman mapping experiments. Fig. 5(a) and (b) illustrate the confocal Raman intensity spatial mapping of multiple foci modification and horizontalpolarization waveguide. The Raman mode intensities show three clear damage zones, which correspond to the spots labeled in Fig. 1. The local reduction in the $\mathrm{TO}_{4}$ mode $\left(\omega=600 \mathrm{~cm}^{-1}\right)$ phonon mode intensity has been shown of up to $43 \%$. In addition, the $\mathrm{TO}_{4}$ phonon mode normalized intensity profile has been obtained along the longitudinal scan as shown in Fig. 5(b) and (d). Clearly, the periodic reductions of the Raman signal at damage spots are observed. This phenomenon could be associated to high density defects creation in those regions, as a consequence of laser-induced micro-explosions at several high intensity excitation focal volumes caused by self-focusing of ultrafast pulse, which disrupt the crystal structure and leads to a decrease in the Raman intensity. This fact supports the presence of periodic damaged high-intensity spots.

We carried out the refractive index contrast between the damage lines and the waveguide region using the technique developed by Siebenmorgen $e t$ al. By assuming a step-index profile, 


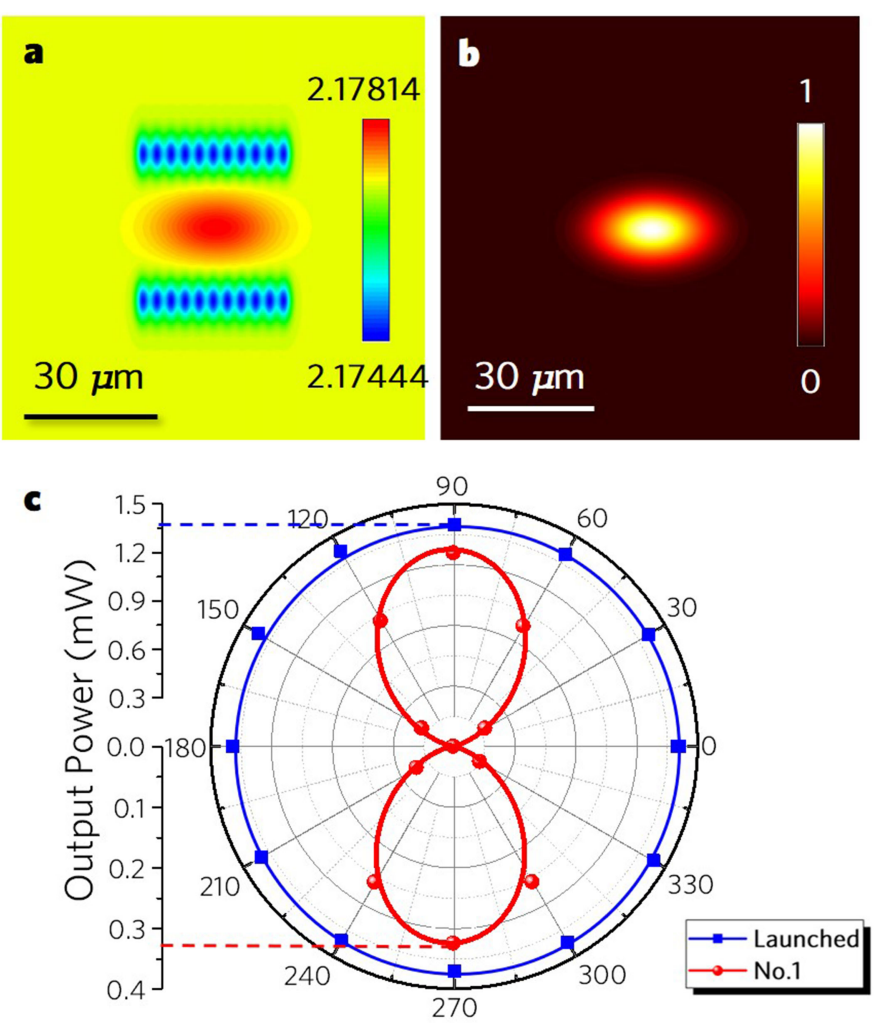

Fig. 6. (a) Reconstructed refractive index profile at the cross section. (b) Calculated modal profile of horizontal dual-line waveguide along TE polarization at $632.8 \mathrm{~nm}$. (c) Output power as a function of all-angle light transmission with the same launched power of waveguide No. 1.

the maximum refractive index change could be roughly estimated via measuring the numerical aperture of the waveguides, using the equation [35]

$$
\Delta n=\frac{\sin ^{2} \theta_{m}}{2 n}
$$

where $\theta_{m}$ is the maximum incident angular deflection at which no transmitted power change occurred, $n$ is the refractive index of LT at $633 \mathrm{~nm}$. In this work, the calculated index changes $\Delta n$ for waveguide No. 1 was estimated to be $3.7 \times 10^{-3}$, along the TE polarization. With this change, the refractive index profile was reconstructed by using the method from the measured near-field intensity introduced by I. Mansour et al. [36]. The numerically calculated near-field profile could be found analytically starting from Maxwell's equations, and was used to calculate the refractive index profile. The distribution of refractive index change could be given by

$$
\Delta n(x, z)=\sqrt{n_{s}^{2}-f \frac{1}{k^{2} A} \nabla_{t}^{2} A}-n_{s}
$$

where $k(=2 \pi / \mathrm{x})$ is the propagation constant, and $A$ is the normalized electric field intensity, which can be calculated

$$
A(x . y)=\sqrt{\frac{I(x, z)}{I_{\max }}}
$$

Fig. 6(a) shows the reconstructed 2D refractive index profile. According to this, the calculated modal profiles has been

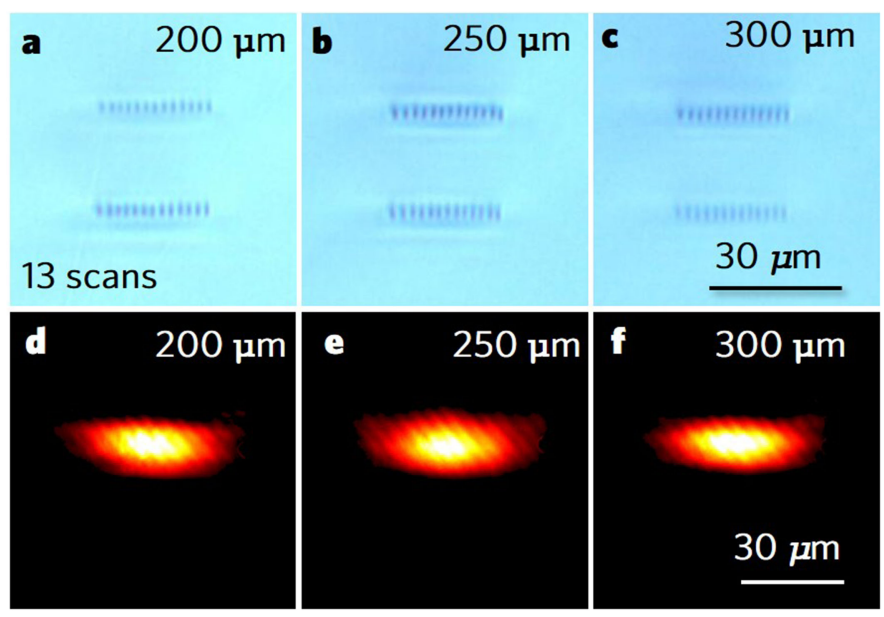

Fig. 7. Optical microscope images of signal-channel at different focal depths, (a) $200 \mu \mathrm{m}$, (b) $250 \mu \mathrm{m}$ and (c) $300 \mu \mathrm{m}$. Measured near-field modal profiles of waveguides for TE polarizations at $633 \mathrm{~nm}$. The pulse energy was fixed at $0.87 \mu \mathrm{J}$.

demonstrated in Fig. 6(b) by using the commercial program BeamPROP (Rsoft, Inc), which is based on the finite-difference beam propagation method (FD-BPM) [37]. The calculated modal profile shows a good agreement with the experimental data. Based on the end-face coupling system, we have estimated propagation losses of the waveguides in MgSLT crystal by using the equation

$$
\alpha=\frac{-10}{L} \log _{10}\left[\frac{P_{\text {out }}}{\eta P_{\text {in }}(1-R)^{2}}\right]
$$

where $P_{\text {in }}$ is the input power, $P_{\text {out }}$ is the output power, for the two-channel waveguides, the output power is the addition of powers of both channels. $L$ is the sample length and $R$ is the reflectance. $\eta$ is the mismatch coefficient, which could be obtained by FD-BPM algorithm (Rsoft Beam PROP). The obtained value for TE modes at $632.8 \mathrm{~nm}$ is $2.9 \mathrm{~dB} / \mathrm{cm}$ and $2.3 \mathrm{~dB} / \mathrm{cm}$ for waveguides No. 1 and No. 2, respectively. For the purpose of investigating the polarization effects of guidance, all-angle light transmission was shown in Fig. 6(c). When polarization angles are $90^{\circ}$ and $270^{\circ}$, corresponding to TE polarization, the output power reaches maximum, showing good guidance for the TE polarization.

In order to investigate the influence of laser parameters, such as laser pulse energy and focal depth on the guiding properties and modal profiles of the waveguides, we first fabricated waveguides at different focal depths with fixed pulse energy. Fig. 7(a)(c) illustrates the optical microscope image of signal-channel at different focal depths with a fixed pulse energy. The corresponding near-field modal profiles are shown in Fig. 7(d)-(f), respectively. The propagation losses of each waveguides at different depth are $1.9 \mathrm{~dB} / \mathrm{cm}, 1.7 \mathrm{~dB} / \mathrm{cm}$ and $2.0 \mathrm{~dB} / \mathrm{cm}$, corresponding to 200, 250 and $300 \mu \mathrm{m}$, respectively. It is observed that there exists an optimal focal depth of $250 \mu \mathrm{m}$. We then fabricated waveguides with different pulse energy at the optimal focal depth of $250 \mu \mathrm{m}$. Fig. 8 shows the influence of pulse energy on the guiding properties of two-channel waveguides. Fig. 8(a)-(e) 


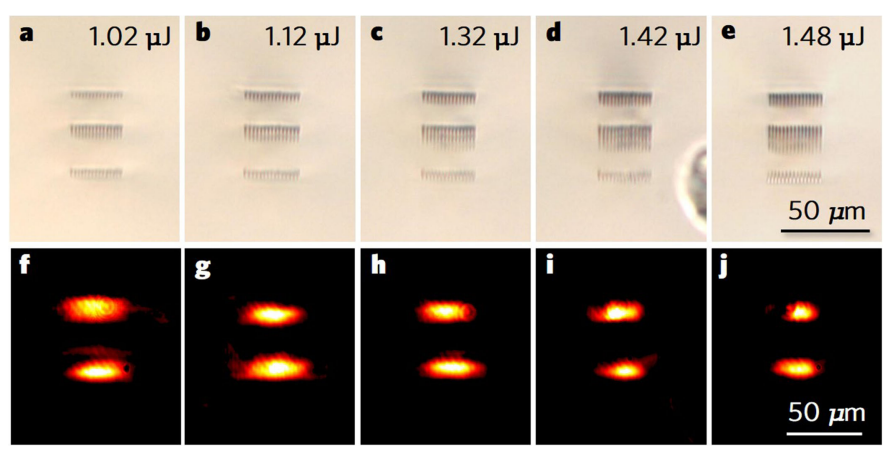

Fig. 8. Optical microscope images of two-channel with different pulse energies of $1.02,1.12,1.32,1.42$ and $1.48 \mu \mathrm{J}$, from left to right, corresponding to (a)-(e), respectively. Measured near-field modal profiles of waveguides for TE polarizations at $633 \mathrm{~nm}$. The focal depth was fixed at $250 \mu \mathrm{m}$.

demonstrate optical microscope image of two-channel with different pulse energies of $1.02,1.12,1.32,1.42$ and $1.48 \mu \mathrm{J}$ at a fixed depth of $250 \mu \mathrm{m}$. The near-field modal profiles are shown in Fig. 8(f)-(j). The propagation losses of each waveguides are $2.5 \mathrm{~dB} / \mathrm{cm}, 2.3 \mathrm{~dB} / \mathrm{cm}, 2.6 \mathrm{~dB} / \mathrm{cm}, 2.9 \mathrm{~dB} / \mathrm{cm}$ and $2.7 \mathrm{~dB} / \mathrm{cm}$. According to this, the optimal pulse energy is $1.12 \mu \mathrm{J}$.

\section{CONCLUSION}

In conclusion, self-formation of aligned multiple foci induced by multiple refocusing of femtosecond pulses in $\mathrm{LiTaO}_{3}$ has been experimentally observed. The number of the high-intensity spots and the period could be controlled by selecting the laser parameters in a desirable way. Based on the spontaneous phenomenon, we have demonstrated the design and one-step fabrication of Type II waveguides, which supported better guidance along TE polarization by implementing multi-scan technique. In particular, one-step two-channel guidance has been realized using our method. Confocal $\mu$-Raman spatial mapping investigations depict the periodic reductions of the Raman signal at the damage spots revealing high density defects creation in those regions. This configuration of horizontal-polarization waveguides by laser writing may also be implemented in other optical crystals thus expanding the potential applications of femtosecondinduced waveguides.

\section{REFERENCES}

[1] D. Kip, "Photorefractive waveguides in oxide crystals: Fabrication, properties, and applications," Appl. Phys. B, vol. 67, no. 2, pp. 131-150, Aug. 1998.

[2] H. Liu, J. R. Vázquez de Aldana, B. del Rosal Rabes, and F. Chen, "Waveguiding microstructures in Nd:YAG with cladding and inner dual-line configuration produced by femtosecond laser inscription," Opt. Mater., vol. 39, pp. 125-129, Jan. 2015.

[3] J. Burghoff, S. Nolte, and A. Tünnermann, "Origins of waveguiding in femtosecond laser-structured $\mathrm{LiNbO}_{3}$," Appl. Phys. A, vol. 89, no. 1, pp. 127132, Jun. 2007.

[4] H. Tang et al., "Experimental two-dimensional quantum walk on a photonic chip," Sci. Adv., vol. 4, no. 5, May 2018, Art. no. eaat3174.

[5] L. Li et al., "All-laser-micromachining of ridge waveguides in $\mathrm{LiNbO}_{3}$ crystal for mid-infrared band applications," Sci. Rep., vol. 7, Aug. 2017, Art. no. 7034

[6] Z. Li, Y. Zhang, C. Cheng, H. Yu, and F. Chen, " 6.5 GHz Q-switched modelocked waveguide lasers based on two-dimensional materials as saturable absorbers," Opt. Express, vol. 26, no. 9, pp. 11321-11330, 2018.
[7] B. B. Xu, Y. L. Zhang, H. Xia, W. F. Dong, H. Ding, and H. B. Sun, "Fabrication and multifunction integration of microfluidic chips by femtosecond laser direct writing," Lab Chip, vol. 13, pp. 1677-1690, Feb. 2013.

[8] K. Sugioka and Y. Cheng, "Ultrafast lasers-Reliable tools for advanced materials processing," Light Sci. Appl., vol. 3, Apr. 2014, Art. no. e149.

[9] T. Meany et al., "Laser written circuits for quantum photonics," Laser Photon. Rev., vol. 9, no. 4, pp. 363-384, Jul. 2015.

[10] Z. N. Tian, J. G. Hua, J. Hao, Y. H. Yu, Q. D. Chen, and H. B. Sun, "Micro-buried spiral zone plate in a lithium niobate crystal," Appl. Phys. Lett., vol. 110, no. 4, Jan. 2017, Art. no. 041102.

[11] Y. L. Zhang, Q. D. Chen, H. Xia, and H. B. Sun, "Designable 3D nanofabrication by femtosecond laser direct writing," Nano Today, vol. 5, no. 5, pp. 435-448, Sep. 2010.

[12] K. M. Davis, K. Miura, N. Sugimoto, and K. Hirao, "Writing waveguides in glass with a femtosecond laser," Opt. Lett., vol. 21, no. 21, pp. 1729-1731, May 1996.

[13] F. Chen and J. R. Vázquez de Aldana, "Optical waveguides in crystalline dielectric materials produced by femtosecond-laser micromachining," Laser Photon. Rev., vol. 8, no. 2, pp. 251-275, May 2014.

[14] M. Xu, R. He, S. Sun, J. R. Vázquez de Aldana, and F. Chen, "Femtosecond laser micromachined optical waveguides in $\mathrm{LiTaO}_{3}$ crystal," Phys. Status Solidi RRL, vol. 7, no. 11, pp. 1014-1017, Aug. 2013.

[15] J. Qi et al., "Fabrication of polarization-independent singlemode waveguides in lithium niobate crystal with femtosecond laser pulses," Opt. Mater. Express, vol. 6, no. 8, pp. 2554-2559, Jul. 2016.

[16] A. L. Stepanov, "Nonlinear optical properties of implanted metal nanoparticles in various transparent matrixes: A review," Rev. Adv. Mater. Sci., vol. 27, pp. 115-145, Feb. 2011.

[17] S. Gross and M. J. Withford, "Ultrafast-laser-inscribed 3D integrated photonics: Challenges and emerging applications," Nanophotonics, vol. 4, pp. 332-352, Jul. 2015.

[18] J. H. Marburger, "Self-focusing: Theory," Prog. Quantum Electron., vol. 4, pp. 35-110, Apr. 1975.

[19] M. Garcia-Lechuga, J. Siegel, J. Hernandez-Rueda, and J. Solis, "Imaging the ultrafast Kerr effect, free carrier generation, relaxation and ablation dynamics of lithium niobate irradiated with femtosecond laser pulses," $J$. Appl. Phys., vol. 116, Sep. 2014, Art. no. 113502.

[20] Z. Wu, H. Jiang, L. Luo, H. Guo, H. Yang, and Q. Gong, "Multiple foci and a long filament observed with focused femtosecond pulse propagation in fused silica," Opt. Lett., vol. 27, no. 6, pp. 448-450, Mar. 2002.

[21] W. Liu, S. L. Chin, O. Kosareva, I. S. Golubtsov, and V. P. Kandidov, "Multiple refocusing of a femtosecond laser pulse in a dispersive liquid (methanol)," Opt. Commun., vol. 225, nos. 1-3, pp. 193-209, Sep. 2003.

[22] D. Ashkenasi, H. Varel, A. Rosenfeld, S. Henz, J. Herrmann, and E. E. B. Cambell, "Application of self-focusing of ps laser pulses for threedimensional microstructuring of transparent materials," Appl. Phys. Lett., vol. 72, no. 12, pp. 1442-1444, Mar. 1998.

[23] E. Toratani, M. Kamata, and M. Obara, "Self-fabrication of void array in fused silica by femtosecond laser processing," Appl. Phys. Lett., vol. 87, Oct. 2005, Art. no. 171103.

[24] S. Kanehira, J. Si, J. Qiu, K. Fujita, and K. Hirao, "Periodic nanovoid structures via femtosecond laser irradiation," Nano Lett., vol. 5, no. 8, pp. 1591-1595, Jul. 2005.

[25] H. Sun et al., "Standing electron plasma wave mechanism of void array formation inside glass by femtosecond laser irradiation," Appl. Phys. A, vol. 88, no. 2, pp. 285-288. Aug. 2007.

[26] S. Sowa, W. Watanabe, J. Nishii, and K. Itoh, "Filamentary cavity formation in poly (methyl methacrylate) by single femtosecond pulse," Appl. Phys. A, vol. 81, no. 8, pp. 1587-1590. Dec. 2005.

[27] $\mathrm{X}$. Hu et al., "Self-formation of void array in $\mathrm{Al}_{2} \mathrm{O}_{3}$ crystal by femtosecond laser irradiation," Chin. Opt. Lett., vol. 6, no. 5, pp. 388-390. May 2008.

[28] X. Hu, Y. Dai, L. Yang, J. Song, C. Zhu, and J. Qiu, "Self-formation of quasiperiodic void structure in $\mathrm{CaF}_{2}$ induced by femtosecond laser irradiation," J. Appl. Phys., vol. 101, no. 2, Jan. 2007, Art. no. 023112.

[29] J. Song, X. Wang, and J. Xu, "Microstructures induced in the bulk of $\mathrm{SrTiO}_{3}$ crystal by a femtosecond laser," Opt. Express, vol. 15, no. 5, pp. 2341-2347, Mar. 2007.

[30] I. Dolev, A. Ganany-Padowicz, O. Gayer, A. Arie, J. Mangin, and G. Gadret, "Linear and nonlinear optical properties of $\mathrm{MgO}: \mathrm{LiTaO}_{3}, "$ Appl. Phys. B, vol. 96, nos. 2/3, pp. 423-432, Aug. 2009.

[31] M. Marangoni, M. Lobino, and R. Ramponi, "High quality buried waveguides in stoichiometric $\mathrm{LiTaO}_{3}$ for nonlinear frequency conversion," Opt. Express, vol. 14, no. 1, pp. 248-253, Jan. 2006. 
[32] W. Ryba-Romanowski, B. Macalik, A. Strzęp, R. Lisiecki, P. Solarz, and R. M. Kowalski, "Spectral transformation of infrared ultrashort pulses in laser crystals," Opt. Mater, vol. 36, no. 10, pp. 1745-1748, Aug. 2014

[33] A. Couairon and A. Mysyrowicz, "Femtosecond filamentation in transparent media," Phys. Rep., vol. 441, nos. 2-4, pp. 47-189, Mar. 2007.

[34] Y. R. Shen, Self-Focusing and Filaments of Light: Past and Present, 3rd ed. New York, NY, USA: Springer, 2009, pp. 3-19.

[35] J. Siebenmorgen, K. Petermann, G. Huber, K. Rademaker, S. Nolte, and A Tünnermann, "Femtosecond laser written stress-induced $\mathrm{Nd}: \mathrm{Y}_{3} \mathrm{Al}_{5} \mathrm{O}_{12}$ (Nd:YAG) channel waveguide laser," Appl. Phys. B, vol. 97, pp. 251-255, Oct. 2009.

[36] I. Mansour and F. Caccavale, "An improved procedure to calculate the refractive index profile from the measured near-field intensity," J. Lightw. Technol., vol. 14, no. 3, pp. 423-428, Mar. 1996.

[37] Computer Software BeamPROP Version 8, Rsoft Design Group, Ossining NY, USA, 2007. [Online]. Available: http://www.rsoftdesign.com

Lingqi Li received the B.A. degree from Shandong Normal University, Jinan, China, in 2016. She is currently working toward the Ph.D. degree under the supervision of Prof. Feng Chen with the School of Physics, Shandong University.

Her current research interests include femtosecond laser micro- and nanofabrication, waveguide lasers, and nonlinear optics.

Weijie Nie received the B.A. degree from Shandong Normal University, Jinan, China, in 2014. She is currently working toward the Ph.D. degree under the supervision of Prof. Feng Chen with the School of Physics, Shandong University.

Her current research interests include laser writing of waveguides, waveguide lasers, and nanoparticles.

Ziqi Li received the B.A. degree from Shandong Normal University, Jinan, China, in 2016. He is currently working toward the Ph.D. degree under the supervision of Prof. Feng Chen with the School of Physics, Shandong University.

His current research interests include ultrafast lasers, nonlinear optics, lowdimensional materials, laser materials processing, and ion beam physics.

Bin Zhang received the B.A. degree from Shandong Normal University, Jinan, China, in 2017. He is currently working toward the M.A. degree under the supervision of Prof. Feng Chen with the School of Physics, Shandong University.

His current research interests include nonlinear optics, femtosecond laser micro- and nanofabrication, and laser material processing.
Lei Wang received the Ph.D. degree from Shandong University, Jinan, China, in 2010 .

He is currently an Associate Professor with the School of Physics, Shandong University. His research interests include material modifications by ultrafast lasers and ion beam, nonlinear optics, and optical waveguides.

Patricia Haro-González received the Ph.D. degree in physics from the Universidad de La Laguna, San Cristóbal de La Laguna, Spain, in May 2011.

In 2013, she joined the Fluorescence Imaging Group, Universidad Autónoma de Madrid, Madrid, Spain, as a Juan de la Cierva Postdoctoral Researcher. In 2018, she became an Assistant Professor with the Physics of Materials Science Department, Universidad Autónoma de Madrid. Currently, her research topics are centered in optical trapping of nanoparticles and their biological application as nanobiosensors.

Daniel Jaque received the "Certificate in Physics" from the University of Sussex, Brighton, U.K., in 1995, and the Ph.D. degree (within Prof. García-Sole's group) from the Universidad Autónoma de Madrid (UAM), Madrid, Spain, in 1999.

He then joined the Universidad Complutense de Madrid, Madrid, as an Assistant Professor. In 2001, he moved back to UAM and rejoined the Laser Spectroscopy Group. His research interests include the properties (optical and magnetic) of nano-structured magnetic thin films, micro/nano structuration of optical materials by ultrafast laser inscription, and confocal fluorescence imaging techniques at the femtosecond time scale. He was the recipient of the "Young Researcher Award" by the European Association for the Study of Rare Earths and Actanides in 2006

Javier R. Vázquez de Aldana received the Bachelor of Science and Ph.D. degrees from the University of Salamanca, Salamanca, Spain, in 1997 and 2001, respectively.

He is currently an Associate Professor with the Science Faculty, University of Salamanca. He is a member of the Laser Microprocessing Research Group and also a Technical and Scientific Advisor of the Laser Facility, University of Salamanca. His research interests include the interaction of intense femtosecond pulses with materials and its application to fabrication of photonic devices.

Feng Chen received the Ph.D. degree from Shandong University, Jinan, China, in 2002 .

He is currently a Professor with the School of Physics, Shandong University. From 2003 to 2005, he was with the Clausthal University of Technology, Clausthal-Zellerfeld, Germany, as an Alexander von Humboldt Research Fellow. In 2006, he became a Professor with Shandong University. His research interests include material modifications by ultrafast lasers and ion beams, optical waveguides, 2D materials, plasmonics, nonlinear optics, and lasers. He is a Fellow of the Institute of Physics, U.K., a senior member of the Optical Society of America, and a Director Board Member of the Chinese Physical Society. He also serves as an Editorial Board Member of Optical Engineering, Scientific Reports, and Chinese Optics Letters. 